\title{
EDITORIAL
}

\section{Heart biomarkers as prognostic tools for chronic thromboembolic pulmonary hypertension: a step forward by the fatty acid-binding protein}

\author{
S.E. Orfanos* and S. Zakynthinos ${ }^{\#}$
}

D uring the last $20 \mathrm{yrs}$, a tremendous amount of scientific discoveries have greatly improved our knowledge on the pathophysiology of pulmonary hypertension, and expanded our armamentarium of important novel medications that have substantially improved survival and the quality of life of patients suffering from this devastating disorder. Although most investigations have focused on pulmonary arterial hypertension $(\mathrm{PAH})$, chronic thromboembolic pulmonary hypertension (CTEPH) has also gained increasing attention [1]. CTEPH could be viewed as an extension of the natural history of acute pulmonary embolism (PE). However, its pathophysiology is not entirely understood; only a minority of patients who have undergone acute PE will develop chronic thromboembolic disease, while disorders associated with abnormal fibrinolysis and/or a pro-thrombotic lung vascular phenotype appear to be predisposing factors for CTEPH development [2].

Chronic thromboembolic pulmonary arterial obstruction will increase pulmonary vascular resistance, subsequently leading to right heart failure and, if untreated, to death $[1,3]$. It has been suggested that in addition to organised thromboemboli, structural remodelling of the pre-capillary vessels, such as in $\mathrm{PAH}$, may also occur in CTEPH further contributing to the haemodynamic compromise of the patient [4]. Organised thrombi located in central proximal vessels can be removed by means of pulmonary thrombo-endarterectomy (PEA) and might lead to phenomena of patients who had been in World Health Organization functional class IV pre-operatively, and who improve to functional class II, or even I, post-operatively. Patients with either distal or inoperable disease, or persistent pulmonary hypertension post-PEA are candidates for treatment with agents used in PAH [3,5]. PEA is usually performed in specialised centres, is relatively expensive and carries a perioperative mortality that could still be $>10 \%$, depending on the operative centre [6]. Thus, it is apparent that markers that identify patients who would benefit the most from surgery, as

\footnotetext{
*Second Dept of Critical Care, University of Athens Medical School and Pulmonary Hypertension Clinic, Attikon Hospital, and ${ }^{\#}$ First Dept of Critical Care and Pulmonary Services, Evangelismos Hospital, University of Athens Medical School, Athens, Greece.
}

STATEMENT OF INTEREST: None declared.

CORRESPONDENCE: S.E. Orfanos, Second Dept of Critical Care, Attikon Hospital, 1, Rimini St, 124 62, Haidari-Athens, Greece. Fax: 30 2105326414. E-mail: sorfanos@med.uoa.gr well as allowing monitoring of the effect of medical treatment on disease progress, are of great value.

Optimal biomarkers should be sensitive, specific and provide quantification of the underlying pulmonary vascular disease and/or right heart dysfunction [7]. In addition, they should be noninvasive, inexpensive and able to offer predictive information on the best possible treatment for patients (i.e. medical or surgical) and outcome. Despite the fact that several compounds, such as the best studied natriuretic peptides in plasma, have already been used along with parameters related to pulmonary haemodynamics and patients' functional performance [7], the need for optimal biomarkers is always present.

In this issue of the European Respiratory Journal, LANKEIT et al. [8] have validated the prognostic value of heart-type fatty acidbinding protein (H-FABP) in CTEPH. Fatty acid-binding proteins (FABPs) are relatively small (15 kDa) cytosolic proteins that are widely distributed and highly expressed in tissues undergoing active fatty-acid metabolism, such as the heart and the liver [9]. FABPs exhibit a high affinity for the noncovalent binding of fatty acids and are named after the tissue from which they are initially identified. The H-FABP isoform is one of the most abundant proteins in the heart, representing $5-15 \%$ of the whole aqueous cytosolic protein pool. H-FABPs are truly cytosolic and cannot been found outside the cell under normal conditions [10]. The fact that $\mathrm{H}-$ FABP is a small protein, allows for rapid diffusion outside the injured myocardium and subsequently rapid detection on plasma post-myocardial injury [9]. All the aforementioned properties suggest strong theoretical and practical advantages for the use of $\mathrm{H}-\mathrm{FABP}$ in detecting myocardial injury.

Several recent studies have provided evidence on the validity of $\mathrm{H}$-FABP for the diagnosis and risk stratification in acute coronary syndromes, as well as the advantage of this novel biomarker over compounds such as troponin and brain natriuretic peptide (BNP) [11-13]. Besides its use in acute coronary syndromes, the validity of this novel biomarker has been additionally demonstrated in chronic heart decompensation. Patients with advanced chronic heart failure have increased serum levels of H-FABP that persist over time in subjects at high risk for adverse clinical outcomes, in contrast to the concomitant BNP decrease [14].

In addition to the study by LANKEIT et al. [8] on the use of $\mathrm{H}$ FABP in CTEPH [8], PULs et al. [15] have validated the use of 
this novel biomarker in risk stratification of patients with acute PE. PULS et al. [15] prospectively studied 107 patients for PErelated death or major complications and overall 30-day mortality. Serum H-FABP on admission, along with Nterminal pro-BNP (NT-proBNP) and cardiac troponin, were compared by means of multivariate logistic regression analysis. H-FABP was identified as a strong independent predictor of the aforementioned study end-points, in contrast with the $\mathrm{N}$-terminal pro-BNP (NT-proBNP) and cardiac troponin, which revealed no significant predictivity. H-FABP remained a superior prognostic indicator even when compared with the maximal NT-proBNP and troponin levels over the first $24 \mathrm{~h}$ post-patient admission [15]. The superiority of H-FABP over troponin and myoglobin for risk stratification in acute PE has also been shown by KACZYNSKA et al. [16]. The fact that H-FABP appears to be an early and promising indicator of right ventricular dysfunction and injury, following acute lung vascular decompensation, has provided a strong rationale for investigating its potential usefulness in CTEPH.

LANKEIT et al. [8] studied 93 consecutive patients with CTEPH and investigated the value of H-FABP as a predictor of adverse outcome, defined as CTEPH-related death, lung transplantation or persistent pulmonary hypertension after PEA. Baseline H-FABP levels in plasma ranged $0.69-24.3 \mathrm{ng} \cdot \mathrm{mL}^{-1}$ and were weakly correlated with cardiac output, mean right atrial pressure, pulmonary capillary wedge pressure and 6-min walking distance. Levels were significantly higher in patients with an adverse outcome, while a univariable Cox regression analysis revealed a hazard ratio of 1.10 for each increase of $\mathrm{H}$ FABP by $1 \mathrm{ng} \cdot \mathrm{mL}^{-1}$. Additional multivariate analysis revealed that H-FABP was an independent predictor of adverse outcome, along with mean right atrial pressure and PEA (with PEA being associated with a lower risk of adverse outcome). It is noteworthy that all other pulmonary haemodynamic parameters, including cardiac output, did not exhibit significant predictivity at multivariate analysis, further supporting the greater prognostic value of this new biomarker. Cardiac troponin $\mathrm{T}$ was additionally measured but, in contrast with a previous report on patients with pre-capillary pulmonary hypertension of diverse aetiologies [17], it was detected in only four patients, indicating a superiority of H-FABP in relation to cardiac troponin $\mathrm{T}$ in stratifying risk in CTEPH. However, it should be mentioned that all four patients had an adverse outcome, suggesting that cardiac troponin T elevation appears, when present, to be an ominous prognostic indicator in CTEPH.

Although the findings mentioned previously already add substantial novel information to our knowledge of CTEPH, perhaps the major finding of the study by LANKEIT et al. [8] is related to the identified value of H-FABP as an outcome predictor in patients who underwent PEA. Indeed, LANKEIT et al. [8] further analysed the data obtained from the 52-patient subgroup that underwent surgery. They showed that subjects with an adverse long-term outcome had significantly higher baseline levels of H-FABP than patients carrying a favourable outcome. This finding was further supported by Kaplan-Meier analysis showing that surgically treated patients with H-FABP plasma values $>2.7 \mathrm{ng} \cdot \mathrm{mL}^{-1}$ (i.e. the median value in the surgically treated cohort) at diagnosis had a lower probability of event-free survival post-PEA. Therefore, it is probable that H-FABP estimations may help identify CTEPH patients who will benefit the most from surgical treatment. Future large prospective studies should be performed to further validate this finding. Studies that will include sequential measurements of H-FABP could additionally validate its use as a marker for monitoring and probable quantification of patients' responses to medical and/or surgical treatment.

As already acknowledged by the authors, two issues were not addressed in the study by LANKEIT et al. [8]. First, the prognostic value of H-FABP was not compared with that of the natriuretic peptides BNP and NT-proBNP, which are commonly used as biomarkers in pulmonary hypertension assessment [7]. In this respect, REESINK et al. [18] have recently shown that plasma BNP levels in CTEPH patients correlated with right ventricular remodelling, as revealed by cardiac magnetic resonance imaging, and could identify right ventricular dysfunction. Studies combining and comparing measurements of H-FABP with those of additional biomarkers in use are needed.

The second issue is related to the fact that patients receiving treatment with novel agents originally reserved for pulmonary arterial hypertension were not included in the study by LANKEIT et al. [8]. Epoprostenol and prostacyclin analogues, the dual endothelin-1 receptor antagonist bosentan, and the phosphodiesterase- 5 inhibitor sildenafil have been shown to be efficacious in chronic thromboembolic pulmonary hypertension patients $[5,6]$. Such pharmacotherapy is now part of the current paradigm of chronic thromboembolic pulmonary hypertension management as a "therapeutic bridge" to pulmonary thrombo-endarterectomy, in inoperable patients, or in persistent pulmonary hypertension post-pulmonary thrombo-endarterectomy. It is likely that future studies will prove that heart-type fatty acid-binding protein is useful in choosing, monitoring or modifying chronic thromboembolic pulmonary hypertension pharmacotherapy.

\section{REFERENCES}

1 Lang IM. Chronic thromboembolic pulmonary hypertension: not so rare after all. N Engl J Med 2004; 350: 2236-2238.

2 Auger WA, Kerr KM, Kim NHS, Ben-Yehuda O, Knowlton KU, Fedullo PF. Chronic thromboembolic pulmonary hypertension. Cardiol Clin 2004; 22: 453-466.

3 Hoeper MM, Mayer E, Simonneau G, Rubin LJ. Chronic thromboembolic pulmonary hypertension. Circulation 2006; 113: 2011-2020.

4 Moser KM, Bloor CM. Pulmonary vascular lesions occurring in patients with chronic major vessel thromboembolic pulmonary hypertension. Chest 1993; 103: 685-692.

5 Bresser P, Pepke-Zaba J, Jais X, Humbert M, Hoeper MM. Medical therapies for chronic thromboembolic pulmonary hypertension. An evolving treatment paradigm. Proc Am Thorac Soc 2006; 3: 594-600.

6 Condliffe R, Kiely DG, Gibbs JS, et al. Improved outcomes in medically and surgically treated chronic thromboembolic pulmonary hypertension. Am J Respir Crit Care Med 2008 [Epub ahead of print PMID: 18292468].

7 Snow JL, Kawut SM. Surrogate end points in pulmonary arterial hypertension: assessing the response to therapy. Clin Chest Med 2007; 28: 75-89.

8 Lankeit M, Dellas C, Panzenböck A, et al. Heart-type fatty acid-binding protein for risk assessment of chronic 
thromboembolic pulmonary hypertension. Eur Respir J 2008; 31: 1024-1029.

9 Pelsers MM, Hermens WT, Glatz JF. Fatty acid-binding proteins as plasma markers of tissue injury. Clinica Chimica Acta 2005; 352: 15-35.

10 Alhadi HA, Fox KA. Do we need additional markers of myocyte necrosis: the potential value of heart fatty acidbinding protein. QJM 2004; 97: 187-198.

11 O'Donoghue $\mathrm{M}$, de Lemos JA, Morrow DA, et al. Prognostic utility of heart-type fatty acid binding protein in patients with acute coronary syndromes. Circulation 2006; 114: 550-557.

12 Kilcullen N, Viswanathan K, Das R, et al. Heart-type fatty acid-binding protein predicts long-term mortality after acute coronary syndrome and identifies high-risk patients across the range of troponin values. J Am Coll Cardiol 2007; 50: 2061-2067.

13 Mad P, Domanovits H, Fazelnia C, et al. Human heart-type fatty-acid-binding protein as a point-of-care test in the early diagnosis of acute myocardial infarction. QJM 2007; 100: 203-210.
14 Niizeki T, Takeishi Y, Arimoto T, et al. Persistently increased serum concentration of heart-type fatty-acidbinding protein predicts adverse clinical outcomes in patients with chronic heart failure. Circ J 2008; 72: 109114.

15 Puls M, Dellas C, Lankeit M, et al. Heart-type fatty acidbinding protein permits early risk stratification of pulmonary embolism. Eur Heart J 2007; 28: 224-229.

16 Kaczynska A, Pelsers MM, Bochowicz A, Kostrubiec M, Glatz JFC, Pruszczyk P. Plasma heart-type fatty acid binding protein is superior to troponin and myoglobin for rapid risk stratification in acute pulmonary embolism. Clinica Chimica Acta 2006; 371: 117-123.

17 Torbicki A, Kurzyna M, Kuca P, et al. Detectable serum cardiac troponin $\mathrm{T}$ as a marker of poor prognosis among patients with chronic precapillary pulmonary hypertension. Circulation 2003; 108: 844-848.

18 Reesink HJ, Tulevski II, Marcus JT, et al. Brain natriuretic peptide as noninvasive marker of the severity of right ventricular dysfunction in chronic thromboembolic pulmonary hypertension. Ann Thorac Surg 2007; 84: 537-543. 